\title{
Dynamic Analysis of an Optimal Active Suspension System Us- ing Global Search Optimization
}

\author{
Muna Khalil Shehan ${ }^{1,2 *}$, B. B. Sahari ${ }^{1}$, Nawal Aswan B. Abdul Jalil ${ }^{1}$, \\ Tang Sai Hong ${ }^{1}$ and Azizan B. As'arry ${ }^{1}$ \\ ${ }^{1}$ Department of Mechanical and Manufacturing Engineering \\ University Putra Malaysia \\ ${ }^{2}$ Ministry of Science and Technology, Baghdad, Iraq \\ *Corresponding author Email: muna.shehan@yahoo.com
}

\begin{abstract}
This paper addresses ride comfort for quarter car active suspension system. Suspension dynamics are modelled by using two degree of freedom vibrating system, linear with time invariant quarter car model to capture the system dynamics when it is subjected to the road disturbance with different velocities. Global search optimization method is a strategy that overcomes the defects of the suspension system performance index formula, objective function (discontinuity, non-smooth) is used to find the optimal suspension spring stiffness and damping coefficient. The optimal active suspension system design is tested when the active elements is malfunctioned. The optimal design is compared with optimal passive suspension system in terms of ride comfort. The results showed that the optimal passive elements of optimal active suspension system provided better ride comfort $\left(0.37 \mathrm{~m} / \mathrm{sec}^{2}\right)$ even at the absent of the active elements compared to optimal passive suspension system.
\end{abstract}

Keywords: active suspension system; ride comfort; optimization.

\section{Introduction}

Vehicle suspension system is an important part in the vehicle. Generally, there are four suspension systems one at each vehicle corner [1], where a suspension system is fitted at each wheel. Suspension system supports the sprung mass (car body) on the unsprung mass (the axles) and has the three main purposes. First, provide good ride comfort through isolating a vehicle body from road disturbances. Typically, ride comfort is measured by the passengers' vertical acceleration [2]. Since ride comfort is subjective issue; hence many experimental studies have proven that it is correlated with the vertical acceleration level and the range of low frequency (1-1.5HZ) [3]. Moreover, suspension system give good handling and road holding on a uneven road, a road disturbance (potholes and bumps), acceleration manoeuvres, braking and lane change. It is not easy to quantify vehicle handling issues due to their subjective nature, but the tire deflection is the easiest quantity that represents road holding [4]. Road holding is connected to the changes in the normal tire forces. Tire force changes are directly connected to tire deflection; hence reducing tire deflection improved braking, traction and cornering. Suspension system supports both the static and dynamic weight of the vehicle. This task is controlled by the suspension rattle space (deflection) and depends on the suspension type used [5]. Vehicle suspension system provide ride comfort by eliminating road irregularities and increase passengers safety by enhancing road holding through regulating the adhesion force between the tires and the road. These demands are mutually conflicting. Ride comfort can be increased at the cost of degraded road holding by using softer suspensions [6]. Suspension design trades these demands off by setting them into weighted performance function for the algorithm of optimization [7]. There are three types of automotive suspension systems; namely passive, conventional, semi active and active suspension systems. Passive suspension system consisted of the uncontrolled spring and damper, shock absorbing. These two components work in parallel and keep supporting the wheel structure and vehicle body. While semi active suspension is a system without active force sources. Thus semi active mechanical layout is similar to passive system, but switching the damper characteristic achieve some control of damping coefficient which gives the ability of damper reaction forces. While active suspension includes an actuator which can provide active force that is controlled by a control algorithm by using data coming from sensors attached to the vehicle. Active suspension system consists of passive elements, spring and damper, and active elements, an actuator. Because of the ability not only to dissipate the energy but also to introduce energy to the system through the actuator, active suspension has the ability to provide the fully accomplish of suspension system aims. However; active suspension controls elements, i.e. sensor and actuator failures are common to any engineered systems. This failure phenomenon can cause total system breakdown (hard failure) or slow degradation i.e. incipient or soft failure. Whatever actuators failure or sensors failure could lead to degradation of suspension performance and cause system instability. Investigation of the active suspension system dynamic properties when the actuator failed provides insights to the dynamic properties of the suspension system passive elements. GS method was suggested to come up with optimal spring stiffness and damping coefficient because it is a powerful method for searching complicated objective function spaces such as Lagrange term to quantify handling and comfort characteristics of the vehicle suspension system where the objec- 
tive function provides increasingly detailed insights into system behaviors.

\section{Active Suspension Model Description}

The active suspension system as shown in Figure 1 was modelled as a linear spring of stiffness $\mathrm{k}_{\mathrm{s}}$ and a linear damper of damping rate $c_{\mathrm{s}}$. The tire is modelled by a spring of stiffness $\mathrm{k}_{\mathrm{t}}$ and the tire damping was neglected due to its small value. Another assumption is that the tire is always in contact with the road surface and does not leave the ground and the sprung mass and unsprung mass vertical displacements $\mathrm{x}_{\mathrm{s}}, \mathrm{x}_{\mathrm{us}}$ are measured from the static equilibrium position. The linearized equation of motion can be derived as follows:

$$
\begin{aligned}
& m_{s} \ddot{x}_{s}+k_{s}\left(x_{s}-x_{u s}\right)+c_{s}\left(\dot{x}_{s}-\dot{x}_{u s}\right)=u \\
& m_{u s} \ddot{x}_{u s}-k_{s}\left(x_{s}-x_{u s}\right)-c_{s}\left(\dot{x}_{s}-\dot{x}_{u s}\right)+k_{t}\left(x_{u s}-x_{o}\right)+ \\
& c_{t}\left(\dot{x}_{u s}-\dot{x}_{o}\right)=-u
\end{aligned}
$$

The desired state variables are chosen to be:

$$
\begin{aligned}
& x_{1}=\left(x_{u s}-x_{o}\right) \\
& x_{2}=x_{u s} \\
& x_{3}=\left(x_{s}-x_{u s}\right) \\
& x_{4}=x_{s}
\end{aligned}
$$

Therefore, eq. (1) and (2) can be rewritten in state space representation eq (7) using the definitions (3)-(6):

$x=A x+b u+D x_{o}$

$\left\{\begin{array}{l}\dot{x}_{1} \\ \dot{x}_{2} \\ \dot{x}_{3} \\ x_{4}\end{array}\right\}=\left[\begin{array}{cccc}0 & 1 & 0 & 0 \\ -\frac{4 k_{t}}{m_{u s}} & -\frac{4\left(c_{s}+c_{t}\right)}{m_{u s}} & \frac{4 k_{s}}{m_{u s}} & \frac{4 c_{s}}{m_{u s}} \\ 0 & -1 & 0 & 1 \\ 0 & \frac{4 c_{s}}{m_{s}} & -\frac{4 k_{s}}{m_{s}} & -\frac{4 c_{s}}{m_{s}}\end{array}\right]\left\{\begin{array}{l}x_{1} \\ x_{2} \\ x_{3} \\ x_{4}\end{array}\right\}+$

$\left\{\begin{array}{c}0 \\ \frac{-1}{m_{u s}} \\ 0 \\ \frac{1}{m_{s}}\end{array}\right\} u+\left\{\begin{array}{c}0 \\ \frac{4 c_{t}}{m_{u s}} \\ 0 \\ 0\end{array}\right\}$

$X_{o}$ is the velocity of the input at the tire, $\mathrm{m}_{\mathrm{s}}, \mathrm{m}_{\mathrm{us}}$ are the sprung mass and unsprung mass respectively. The road input disturbance profile used in the optimization algorithm is based on stochastic road profile (IRI) which is a standard technique for the international roughness index. The block diagram of the state representation equation (7) is shown in Figure 2. Figure 2 shows two inputs to the active suspension system, first is the random elevation profile over the length of the road, and the control force.

The suspension system constant parameters are given in Table 1 that contains all the parameters used in both the optimization algorithm and simulation algorithm.

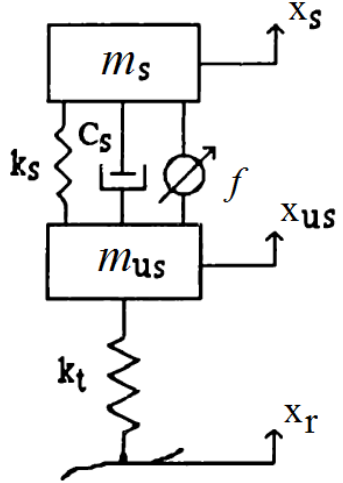

Fig. 1: Quarter car model suspension system

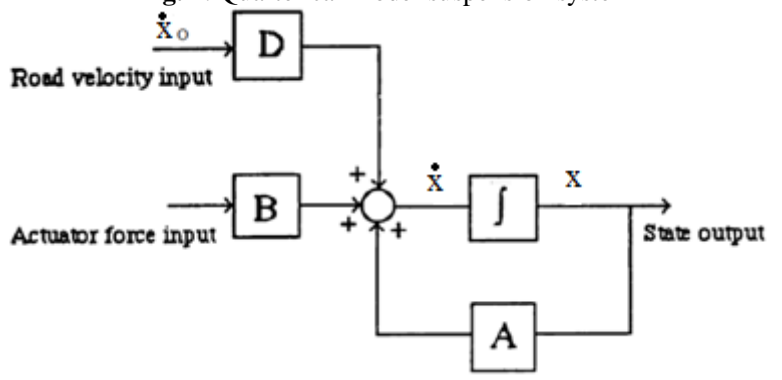

Fig. 2: Block diagram of active suspension system

Table 1: Suspension System Constant Parameters

\begin{tabular}{|c|c|}
\hline parameters & values \\
\hline Sprung mass & $325 \mathrm{~kg}$ \\
\hline Unsprung mass & $65 \mathrm{~kg}$ \\
\hline Tire stiffness & $232 \mathrm{kN} / \mathrm{m}$ \\
\hline Initial spring stiffness & $10000 \mathrm{~N} / \mathrm{m}$ \\
\hline Initial damping coefficient & $1000 \mathrm{~N} . \mathrm{sec} / \mathrm{m}$ \\
\hline
\end{tabular}

\section{Global Search Optimization Algorithm}

Global Search (GS), a strategy used to find the system optimal design variables, is started from a local solver (Fmincon) [8] where the algorithm utilize multiple start points to sample multiple basins of attraction. To understand the strategies the problem in eq (9) was considered that abstracted as:

$\min _{k_{s}, c_{s}} J=\int_{0}^{t_{s}}\left(\rho_{1}\left(\ddot{x_{s}}\right)^{2}+\rho_{2}\left(x_{u s}-x_{o}\right)^{2}+\rho_{3} u^{2}\right) d t$

$\rho_{1}, \rho_{2}, \rho_{3}$ Are the optimization parameters and $\mathrm{J}$ is the system level optimization objective. The suspension system performance index, eq. (9), is based on a standard form used in optimal control due to its ability to quantify the impact of time dependent suspension behavior on the overall system performance. The suspension system was optimized with respect to ride comfort, handling and cost of controlling the suspension. Suspension spring stiffness and damping coefficient are the optimization variables where the set of optimization variables satisfying all system level constraints (boundary conditions). This problem formulation is partitioned into plant and controller optimization sub-problems; however in this study an open loop control is used so the value of control force (u) is changed by imposing explicit bounds on the control force and monitoring the change of the suspension passive parameters based on the suspension performance index. Figure 3 depicts the optimization algorithm flow charts. Suspension system is a dynamic system and usually dynamic system is characterized mathematically by a set of ordinary differential equations, ODE. Specifically the dynamics are described for a simulation time ( )by a system of ordinary differential equations. In the proposed study MatLab function ODE 23 was used to determine the desired state variables. Furthermore to determine the ride comfort in terms of sprung mass acceleration, objective function calls the stat variables derivative function to calculate eq. (7), and with the deter- 
mined sprung mass acceleration, eq. (9) satisfied all the requirement data. When the convergence occurs, GS record the optimal design variables Table 2 . The optimal sprung stiffness and damping coefficient were used to test the optimal suspension system in terms of natural frequency. If the natural frequency of the system is about $1 \mathrm{~Hz}$ to $1.5 \mathrm{~Hz}$, the system is assumed as optimal otherwise the control force is adjusted and the optimization algorithm is rerun. Matlab® was used to develop the optimization algorithm.

Table 2: Global Search Results

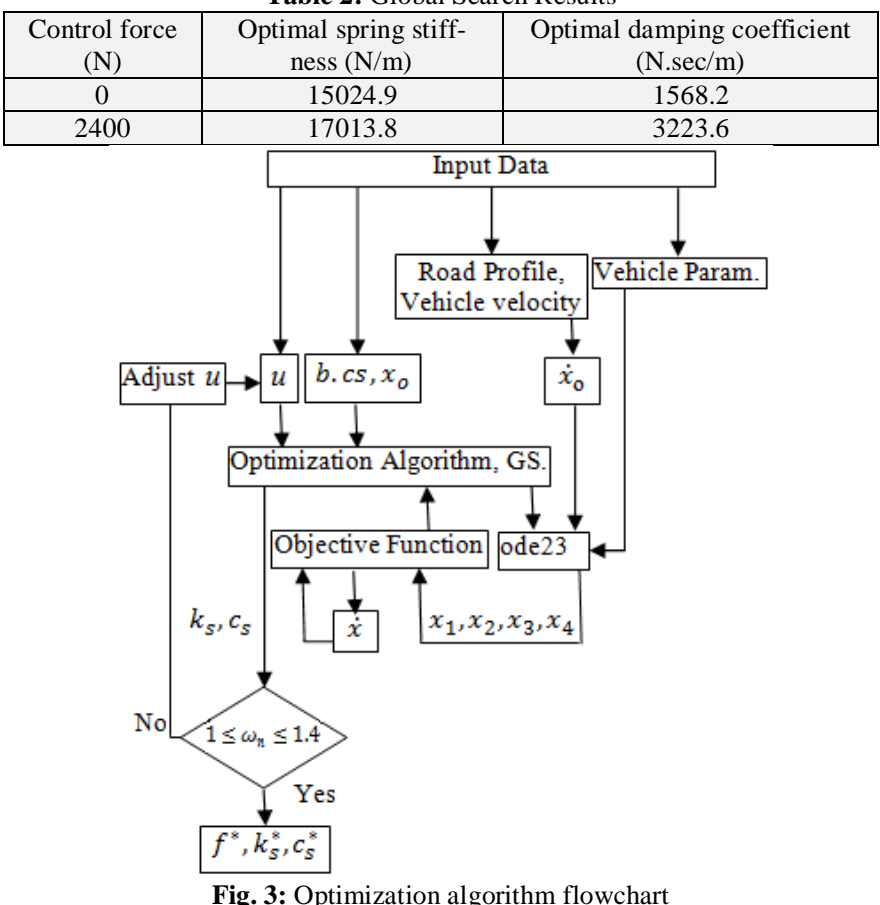

\section{Simulation Algorithm}

Optimization of suspension system (linear state dependent) requires system simulation. The evaluation of associated state derivative is result of the simulation. The suspension system dynamics were analyzed by using different velocities (from $40 \mathrm{~km} / \mathrm{hr}$ to 70 $\mathrm{km} / \mathrm{hr}$ ) when the vehicle travelled at a constant velocity over a speed bump and the suspension system active element was malfunctioned. However; actuator failure is fairly common to any engineered system, where these phenomena may be manifested in breakdown of a system. However; investigation on the suspension passive elements dynamics that are independent of the control system design reflect the suspension dynamic response that has been optimized by the optimization algorithm to work with the active control force, providing insights to the dynamic response of the physical system. The simulation algorithm is started by identifying the input data that contain the vehicle parameters as given in Table 1, speed bump (10 $\mathrm{cm}$ amplitude, $30 \mathrm{~cm}$ width), vehicle velocity that vary from $40 \mathrm{~km} / \mathrm{hr}$ to $70 \mathrm{~km} / \mathrm{hr}$ and the optimization results recorded as the control force vary from $0 \mathrm{~N}$ to $2400 \mathrm{~N}$, the optimization results are a vector of spring stiffness and a vector of damping coefficient. Each optimal design that is one of the optimization algorithm results is converted to a state-space model by using "ss" matlab function that convert the dynamic system to a state space system.

In this work "lsim" which is a Matlab function to simulate time response of dynamic system to arbitrary inputs is used to investigate the dynamic suspension system design problem with computationally derivative function. After created the suspension system state space model with ss function, the bump speed specify the number of states (4 state variables) in the dynamic model and the desired state variables derivative are the output for the model. The simulation algorithm is constricted for only the derivative function (ride comfort) instead of the full system analysis. The algorithm addresses on of the most effective objective of system analysis (vehicle body acceleration). Figure 4 illustrate the simulation algorithm for the optimization results where each optimal design dynamic response was simulated as the vehicle velocity was changed. The simulation results were in terms of the ride comfort and settling time.

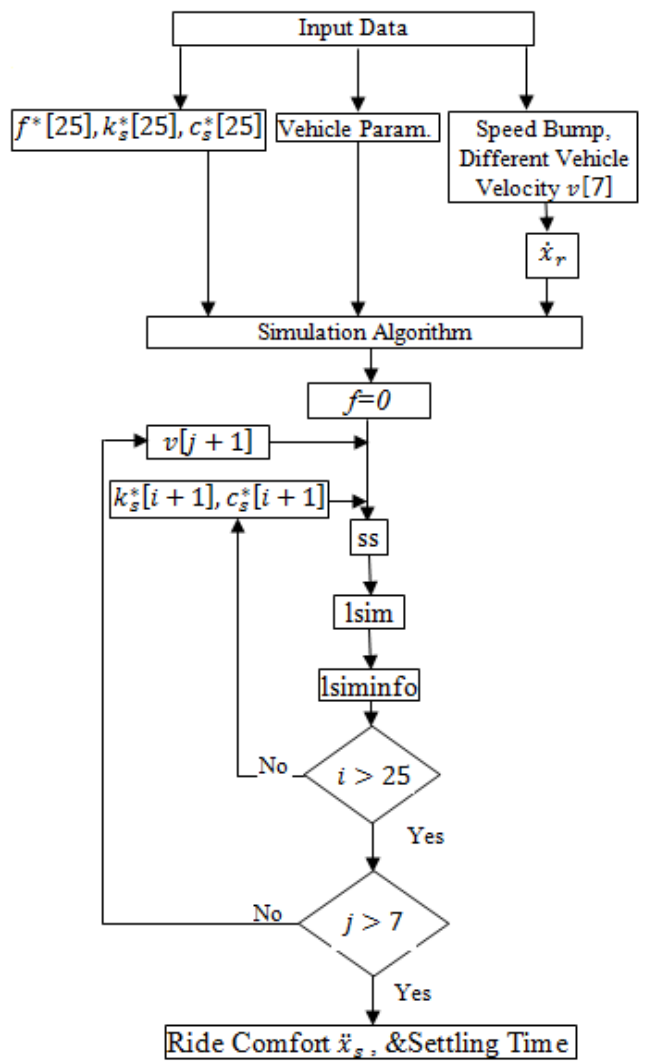

Fig. 4: Simulation Algorithm Flowchart

\section{Results and Discussion}

Global Search was used to find optimal active suspension by multiple runs of a local solver; hence the optimization algorithm was run with varied level of control authority to investigate how the suspension passive elements (spring stiffness and damping coefficient) changed and gave insights to the physical system dynamic properties i.e. natural frequency, damping ratio and settling time. Simulation is often employed to support design decisions and estimate dynamic system performance, and in this study the dynamic response of the vehicle body, sprung mass acceleration was simulated for optimal suspension systems when the control force is $2400 \mathrm{~N}$ and the results were compared with the optimal passive suspension system. For active suspension system, sprung mass acceleration was studied as the effect of the vehicle velocity from $40 \mathrm{~km} / \mathrm{hr}$ to $70 \mathrm{~km} / \mathrm{hr}$ as shown in Figure 5a. Figure 5a illustrated as the vehicle velocity increase, the sprung mass acceleration increase while the settling time decrease due to the effect of damper coefficient. The overshoot of the system is more related to the spring stiffness while the time settling decrease as the vehicle velocity increase this because time settling decrease when the motion ratio decrease where the motion ratio is the damper velocity over the suspension velocity. Low motion ratio calls for a high damping coefficient for the damper itself. Optimal suspension system must reduce the vehicle body acceleration by good isolation the vehicle body from the road roughness. In this study the GS results in terms of optimal sprung stiffness and optimal damping coefficient were integrated in the simulation of quarter car suspension system. Figure $5 \mathrm{~b}$ shows the sprung mass acceleration overshoot of the simulated model as the vehicle velocity increase gradually which is less than $0.315 \mathrm{~m} / \mathrm{sec}^{2}$ that can be considered comfort based on ISO 2361. From Figure 5b, as the vehicle velocity is decreased, the sprung mass acceleration decrease too 
which is mean best ride comfort. The dynamic response in terms of sprung mass acceleration for optimal passive suspension system is shown in Figure $6 a$ as the vehicle velocity was changed, the sprung mass acceleration overshoot and the time settling is illustrated in Figures $6 \mathrm{~b}$ and $6 \mathrm{c}$. A comparison between optimal passive suspension system and optimal active suspension system when the actuator failed in terms of sprung mass acceleration overshoot and settling time was shown in Figure 7. The sprung mass acceleration of proposed active suspension system that integrated the optimal values of spring stiffness and damping coefficient is less than the sprung mass acceleration of the passive suspension system that integrated the GS results even if the control force is absent.

\section{Conclusion}

The optimal numerical values of spring stiffness and damping coefficient for quarter car active suspension system were addressed by proposing a new design methodology, the Global Search (GS) method. Global search optimization algorithm has been developed based on open loop control design. The optimization algorithm integrated model is 2DOF vibrating system, linear with time invariant quarter car suspension system that captures the system dynamics when it is subjected to the road disturbance. Time domain simulation algorithm has been developed. The optimal values of spring stiffness and damping coefficient were integrated in the simulated model of quarter car suspension system to investigate the dynamic response of the proposed model in terms of spring mass acceleration, settling time. The simulation phase results showed that the sprung mass acceleration of the quarter car suspension system that integrated the optimal passive element of the active suspension system is

$\ddot{x}_{s}=0.315 \mathrm{~m} / \mathrm{sec}^{2}$ Which is less than the optimal passive suspension system model of $\ddot{x}_{s}=0.37 \mathrm{~m} / \mathrm{sec}^{2}$. From the results obtained, GS has been proven to be a promising strategy to find the optimal passive elements of active suspension system when the control force is specified directly.
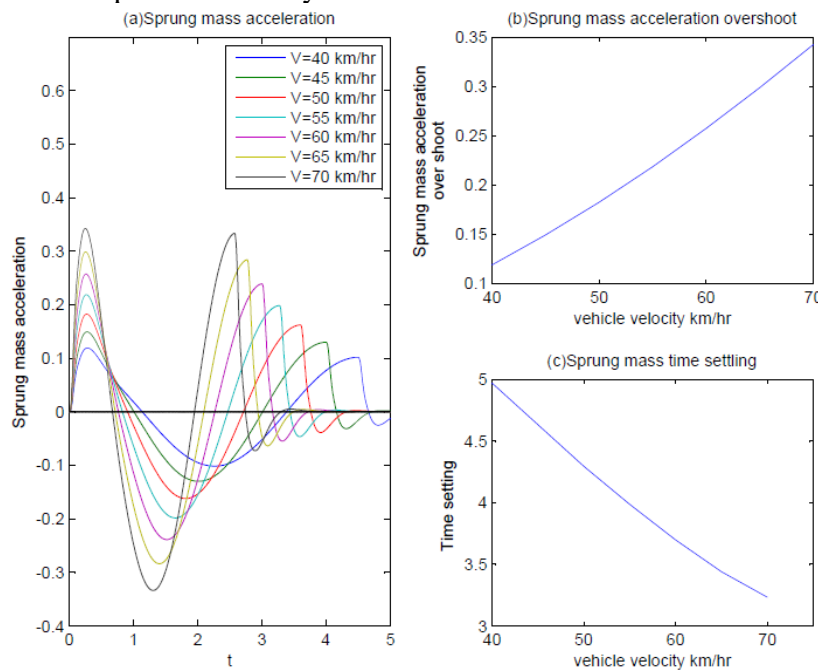

Fig. 5: Sprung mass acceleration for a vehicle travels over a speed bump (10 $\mathrm{cm}$ amplitude and $30 \mathrm{~cm}$ length) where the suspension passive parameters are obtained from the optimization algorithm for $\mathrm{F}=2400 \mathrm{~N}$
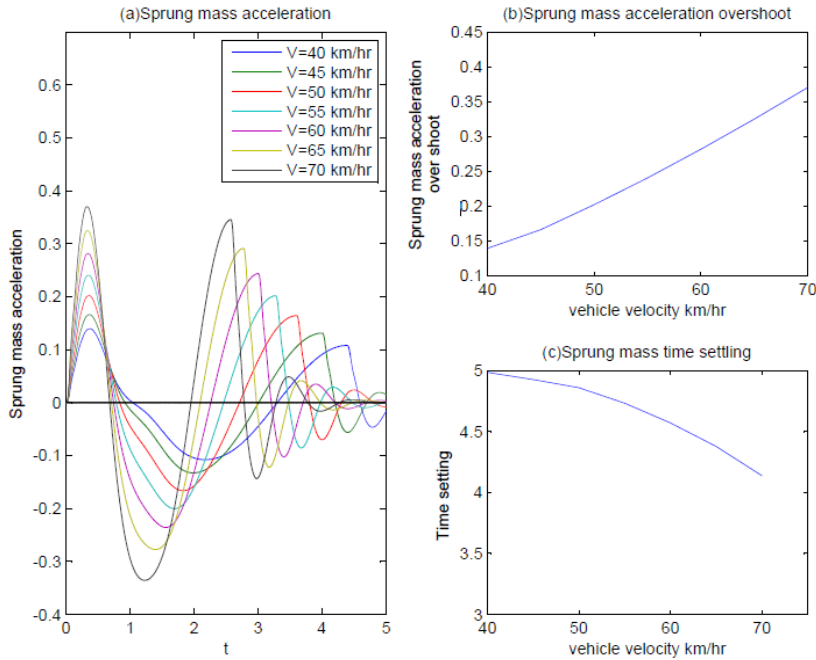

Fig. 6: Sprung mass acceleration for a vehicle travels over a speed bump $(10 \mathrm{~cm}$ amplitude and $30 \mathrm{~cm}$ length) where the suspension passive parameters are obtained from the optimization algorithm for $\mathrm{F}=0 \mathrm{~N}$

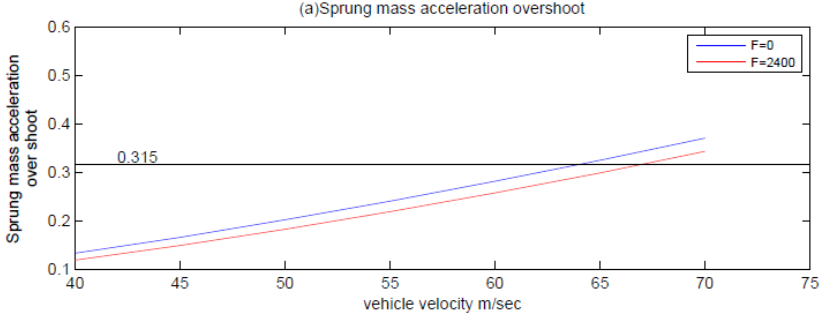

(b)Sprung mass acceleration settling time

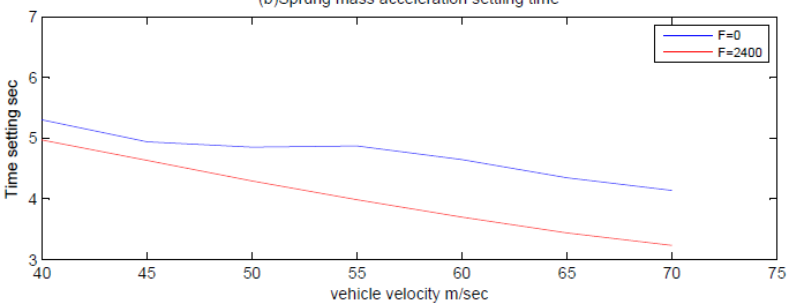

Fig. 6: A comparison between passive suspension system and active suspension system in terms of (a) acceleration overshoot and (b) settling time.

\section{References}

[1] Jazar, Reza N. (2013), Vehicle dynamics: theory and application. Springer Science \& Business Media. New York, NY, United States. ISO (1997), Mechanical Vibration and Shock: Evaluation of Human Exposure to Whole-body Vibration. Part 1, General Requirements: International Standard ISO 2631-1: (E). ISO 1997.

[3] Griffin, Michael J. Handbook of human vibration. 1st Academic press. California, United States. 1996.

[4] Gillespie, Thomas D. (1992), Fundamentals of vehicle dynamics. Society of Automotive Engineers, Inc. Warrendale,Pennsylvania, USA.

[5] Butsuen, Tetsuro (1989), The design of semi-active suspensions for automotive vehicles. PhD diss., Massachusetts Institute of Technology,

[6] Fathy Hosam K, Panos Y, Papalambros A, Galip Ulsoy, \& Davor Hrovat (2003), Nested plant/controller optimization with application to combined passive/active automotive suspensions. American Control Conference, 2003. Proceedings of the vol 4, pp: 3375-3380. IEEE, 2003.

[7] Allison, James T, Tinghao Guo \& Zhi Han (2014), Co-design of an active suspension using simultaneous dynamic optimization. Journal of Mechanical Design 136, no. 8081003.

[8] Coleman T, Branch MA \& Grace A (1999), Optimization toolbox users guide, The MathWorks, Inc. 